\title{
Asymptotic Stability of Delay-Controlled Nonlinear Stochastic Systems with Actuator Failures
}

\author{
N. Zhou' ${ }^{1}$ and R. H. Huan ${ }^{2}$ \\ ${ }^{1}$ State Key Laboratory of Traction Power, Southwest Jiaotong University, Chengdu 610031, China \\ ${ }^{2}$ Department of Mechanics, Key Laboratory of Soft Machines and Smart Devices of Zhejiang Province, \\ Zhejiang University, Hangzhou 310027, China \\ Correspondence should be addressed to R. H. Huan; rhhuan@zju.edu.cn
}

Received 1 April 2017; Accepted 13 June 2017; Published 16 July 2017

Academic Editor: Francesco Ripamonti

Copyright (c) 2017 N. Zhou and R. H. Huan. This is an open access article distributed under the Creative Commons Attribution License, which permits unrestricted use, distribution, and reproduction in any medium, provided the original work is properly cited.

\begin{abstract}
The problem of asymptotic stability of delay-controlled nonlinear stochastic systems with actuator failures is investigated in this paper. Such a system is formulated as a continuous-discrete hybrid system based on the random switch model of failure-prone actuator. Time delay control force is converted into delay-free one by randomly periodic characteristic of the system. Using limit theorem and stochastic averaging, an approximate formula for the largest Lyapunov exponent of the original system is then derived, from which necessary and sufficient conditions for asymptotic stability are obtained. The validity and utility of the proposed procedure are demonstrated by using a stochastically driven nonlinear two-degree system with time delay feedback and actuator failure.
\end{abstract}

\section{Introduction}

Modern technological systems rely on sophisticated control systems to meet increased performance requirements. In such systems, control components (e.g., actuator or sensor) are often subjected to random failures $[1,2]$. It is known that control component failures can cause performance deterioration of control systems or even system instability leading to catastrophic accidents. Therefore, stability analysis for systems with control component failures has been an important and challenging research problem.

In practical applications, actuators are very important in transferring the controller output to the structure, and actuator failures are the typical faults appearing in control systems. In the last few decades, issues concerning stability and control problem of systems with actuator failures have been examined in the literatures. Mahmoud et al. [3] analyzed the stochastic stability of fault tolerant control systems in the presence of noise using the Lyapunov function approach. Gundes [4] studied the stability of the MIMO feedback system under possible failures in either the sensor-connections or actuator-connections. Aberkane et al. [5] dealt with the problem of robust $H_{\infty}$ control of active fault tolerant control systems with Markovian parameters via dynamic output feedback. A state feedback output tracking adaptive control scheme which is developed for plants with actuator failures is proposed by Tao et al. [6]. Seo and Kim [7] designed a robust and reliable $H_{\infty}$ control for linear uncertain systems with actuator failures. The previous studies focused mainly on the linear systems or the one-dimensional nonlinear system. Furthermore, time delay is assumed to be ignorable. However, the phenomenon of time delay is common in control systems, which is a source of instability [8-10]. Thus, development of methodology for the stability analysis of delay-controlled nonlinear systems with actuator failures is thus much deserving.

In the present research, asymptotic stability of delaycontrolled nonlinear systems with actuator failures will be investigated. The organization of this paper is as follows. In the next section, the random switch model of failureprone actuator is established. Section 3 converts time delay control force into the delay-free one using randomly periodic characteristic of the system. Reduced averaged equations are obtained in Section 4. Section 5 gives the necessary and 
sufficient conditions for asymptotic stability of the system. An illustrated example is presented in Section 6, and conclusion follows thereafter.

\section{Formulation of Problem}

2.1. Model of Possible Failure Actuator. The behavior of a possible failure actuator in a control system can be described as

$$
u^{a}(t)=\kappa u(t)
$$

where $u(t)$ is commanding signal to the actuator and $u^{a}$ is the actual output of the possible failure actuator. $\kappa \in$ $\{0,1\}$ is the failure factor which expressed changed actuator characteristics. The case $\kappa=0$ corresponds to complete failure of the actuator and therefore no output; $\kappa=1$ refers to a functional actuator which is working normally. Therefore, in a real control system, the actuator may have two states: a "functional" state and a "failure" state. The "functional" actuator may change to failure actuate due to occasional breakdowns and the failure actuator can also be recovered during a finite time interval due to either efficient mobile maintenance or full redundancy. The random switches between these two states occur as a continuous-time Markov process $[3,5,11]$. Then, (1) can be rewritten in the following Markov jump form:

$$
u^{a}(t)=\kappa^{[s(t)]} u(t)
$$

where $s(t)$ is a Markov process, which points to state of the actuator, and it takes discrete values from a finite set $S=$ $\{1,2\}$. The transition rate from the functional state to failure state is assumed to be $\lambda_{1}$ and the backward transition rate is $\lambda_{2}$. Then, the transition rate matrix $\Lambda$ of $s(t)$ can be specified by $[12]$

$$
\Lambda=\left[\begin{array}{cc}
-\lambda_{1} & \lambda_{1} \\
\lambda_{2} & -\lambda_{2}
\end{array}\right]
$$

Denote by $\mu^{(u)}$ the stationary or equilibrium probability that $s(t)=u(u=1,2)$. Then $\mu^{(u)}$ satisfies the following equations [13]:

$$
\begin{aligned}
\sum_{u=1}^{2} \mu^{(u)} \lambda_{u i} & =0, \\
\sum_{u=1}^{2} \mu^{(u)} & =1,
\end{aligned}
$$

$\mu^{(u)}$ will be used in the next section.

2.2. Nonlinear Stochastic Systems with Actuator Failures. Consider an $n$ degree-of-freedom (DOF) controlled stochastically driven nonlinear system with $n$ actuators marked as $\Omega=\{1,2, \ldots, n\}$. The actuators in the system are distinguished in two groups with regard to potential failures. One group is the set of actuators that are susceptible to failure, denoted by $\Omega_{f}$. The other is a set of actuators that are robust to faults eventually, by $\Omega_{n}=\Omega-\Omega_{f}$. The motion of the system is usually governed by $n$ second-order stochastic differential equations in the generalized displacements. These second-order equations can always be recast as $2 n$ first-order equations in the Hamiltonian formulation

$$
\begin{aligned}
\dot{q}_{i}= & \frac{\partial \widetilde{H}^{\prime}}{\partial p_{i}}, \\
\dot{p}_{i}= & -\frac{\partial \widetilde{H}^{\prime}}{\partial q_{i}}-\varepsilon \widetilde{c}_{i j}^{\prime}(\mathbf{q}, \mathbf{p}) \frac{\partial \widetilde{H}^{\prime}}{\partial p_{j}}+\varepsilon \kappa_{i}^{[s(t)]} u_{i}\left(\mathbf{q}_{\tau}, \mathbf{p}_{\tau}\right) \\
& +\varepsilon^{1 / 2} f_{i k}(\mathbf{q}, \mathbf{p}) W_{k}(t),
\end{aligned}
$$

where $i, j=1,2, \ldots, N, k=1,2, \ldots, m$, and $q_{i}$ and $p_{i}$ are the generalized displacements and momenta, respectively; $\mathbf{q}=$ $\left(q_{1}, \ldots, q_{n}\right)^{\mathrm{T}}$ and $\mathbf{p}=\left(p_{1}, \ldots, p_{n}\right)^{\mathrm{T}} . \varepsilon$ is a small parameter. $\widetilde{H}^{\prime}=\widetilde{H}^{\prime}(\mathbf{q}, \mathbf{p})$ is the Hamiltonian. $-\varepsilon \widetilde{c}_{i j}^{\prime}(\mathbf{q}, \mathbf{p}) \partial \widetilde{H} / \partial p_{j}$ and $\varepsilon^{1 / 2} f_{i k}(\mathbf{q}, \mathbf{p}) W_{k}(t)$ represent the weak damping and pure parametric random excitations, respectively. $W_{k}(t)$ are independent Gaussian white noises with zero means and correction functions $E\left[W_{k}(t) W_{l}(t+\tau)\right]=2 D_{k l} \delta(\tau) \cdot \mathbf{q}_{\tau}=\mathbf{q}(t-\tau)$ and $\mathbf{p}_{\tau}=\mathbf{p}(t-\tau)$ are system's states with time delay. $\kappa_{i}^{[s(t)]}$ are the two-state failure factors

$$
\begin{aligned}
& \kappa_{i}^{(1)}= \begin{cases}1, & i \in \Omega_{n}, \\
0, & i \in \Omega_{f},\end{cases} \\
& \kappa_{i}^{(2)}=1, \quad i \in \Omega .
\end{aligned}
$$

Note that, by modeling the transition between two states of the failure-prone actuator as a Markov jump process, the stochastic stability of system (5a) and (5b) can be studied in the framework of Markov jump hybrid systems.

\section{Randomly Periodic Solution Approximation}

Equations (5a) and (5b) can be converted to the following Itô equations by adding Wong-Zakai correction terms:

$$
\begin{aligned}
d q_{i} & =\frac{\partial \widetilde{H}}{\partial p_{i}} d t \\
d p_{i} & =\left[-\frac{\partial \widetilde{H}}{\partial q_{i}}-\varepsilon \widetilde{c}_{i j}(\mathbf{q}, \mathbf{p}) \frac{\partial \widetilde{H}}{\partial p_{j}}\right. \\
& \left.+\varepsilon \kappa_{i}^{[s(t)]} u_{i}[\mathbf{q}(t-\tau), \mathbf{p}(t-\tau)]\right] d t \\
& +\varepsilon^{1 / 2} \sigma_{i k}(\mathbf{q}, \mathbf{p}) d B_{k}(t),
\end{aligned}
$$

where $\widetilde{H}$ and $\widetilde{c}_{i j}(\mathbf{q}, \mathbf{p})$ are the modified Hamiltonian and coefficients of damping, respectively; $B_{k}(t)$ are standard Wiener processes such that $\sigma_{i k}(\mathbf{q}, \mathbf{p}) d B_{k}(t)=f_{i k}(\mathbf{q}, \mathbf{p}) W_{k}(t) d t$. 
Here, we only consider the case that the associated Hamiltonian system of system (7a) and (7b) is integrable [14]. Then, the Hamiltonian $\widetilde{H}$ has the form

$$
\begin{aligned}
\widetilde{H}(\mathbf{q}, \mathbf{p}) & =\sum_{i=1}^{n} \widetilde{H}_{i}\left(q_{i}, p_{i}\right), \\
\widetilde{H}_{i}\left(q_{i}, p_{i}\right) & =\frac{1}{2} p_{i}^{2}+U_{i}\left(q_{i}\right),
\end{aligned}
$$

where $\widetilde{H}_{1}, \widetilde{H}_{2}, \ldots, \widetilde{H}_{n}$ are $n$ independent first integrals of the associated Hamiltonian system.

For the case of small time delay, it is proved that the following approximate expressions hold [10]:

$$
\begin{aligned}
& q_{i}(t-\tau) \approx \cos \left(\omega_{i} \tau\right) q_{i}(t)-\frac{\sin \left(\omega_{i} \tau\right)}{\omega_{i}} p_{i}(t), \\
& p_{i}(t-\tau) \approx \cos \left(\omega_{i} \tau\right) p_{i}(t)+\omega_{i} \sin \left(\omega_{i} \tau\right) q_{i}(t),
\end{aligned}
$$

where $\omega_{i}$ is the average frequency.

According to expression (9), the time delayed control forces $\kappa_{i}^{[s(t)]} \mathcal{u}_{i}[\mathbf{q}(t-\tau), \mathbf{p}(t-\tau)]$ can be expressed in terms of current state variables with time delay as a parameter; that is, $\kappa_{i}^{[s(t)]} u_{i}[\mathbf{q}(t-\tau), \mathbf{p}(t-\tau)]=\kappa_{i}^{[s(t)]} u_{i}(\mathbf{q}, \mathbf{p} ; \tau)$. Then, splitting $\kappa_{i}^{[s(t)]} u_{i}(\mathbf{q}, \mathbf{p} ; \tau)$ into conservative part and dissipative part and combining them with $-\partial \widetilde{H} / \partial q_{i}$ and $-\varepsilon \widetilde{c_{i j}}(\mathbf{q}, \mathbf{p}) \partial \widetilde{H} / \partial p_{j}$, respectively, yield

$$
\begin{aligned}
& d q_{i}=\frac{\partial H^{\prime[s(t)]}(\tau)}{\partial p_{i}} d t, \\
& d p_{i} \\
& =\left[-\frac{\partial H^{\prime[s(t)]}(\tau)}{\partial q_{i}}-\varepsilon c_{i j}^{\prime[s(t)]}(\mathbf{q}, \mathbf{p} ; \tau) \frac{\partial H^{\prime[s(t)]}(\tau)}{\partial p_{j}}\right] d t \\
& \quad+\varepsilon^{1 / 2} \sigma_{i k}(\mathbf{q}, \mathbf{p}) d B_{k}(t),
\end{aligned}
$$

where $H^{\prime[s(t)]}$ and $c_{i j}^{\prime[s(t)]}$ are the Markov jump Hamiltonian and damping coefficients. Since the control forces are weak, it is reasonable to assume that the conservative part of the delayed control forces may change the Hamiltonian slightly but not change the integrability of the associated Hamiltonian system. Then, the resulting system (10a) and (10b) is still a quasi integrable Hamiltonian system.

\section{Averaged Equations}

According to the limit theorem [15], under the assumption of ergodicity of $s(t)$, the solution of (10a) and (10b) converges in probability of the solution of the following weighted averaging equations as $\varepsilon \rightarrow 0$ :

$$
d q_{i}=\frac{\partial H(\tau)}{\partial p_{i}} d t
$$

$$
\begin{aligned}
d p_{i} & =\left[-\frac{\partial H(\tau)}{\partial q_{i}}\right. \\
& \left.-\varepsilon \sum_{u=1,2}\left[c_{i j}^{\prime(u)}(\mathbf{q}, \mathbf{p} ; \tau) \cdot \mu^{(u)}\right] \frac{\partial H(\tau)}{\partial p_{j}}\right] d t \\
& +\varepsilon^{1 / 2} \sigma_{i k}(\mathbf{q}, \mathbf{p}) d B_{k}(t),
\end{aligned}
$$

where $\mu^{(u)}$ is given in (4), and

$$
\begin{gathered}
H(\tau)=\sum_{i=1}^{n} H_{i}\left(q_{i}, p_{i} ; \tau\right), \\
H_{i}\left(q_{i}, p_{i} ; \tau\right)=\sum_{u=1,2}\left[H_{i}^{\prime(u)}\left(q_{i}, p_{i} ; \tau\right) \cdot \mu^{(u)}\right] .
\end{gathered}
$$

It is noted from (12) that limiting theorem will not change the integrality of the quasi Hamiltonian system. Further assume that the associated Hamiltonian system is nonresonant. Then, by using the stochastic averaging method for the quasi integrable Hamiltonian systems [14], the following averaged Itô equations associated with (11a) and (11b) can be obtained:

$$
d H_{i}=m_{i}(\mathbf{H} ; \tau) d t+\bar{\sigma}_{i k}(\mathbf{H} ; \tau) d B_{k}(t),
$$

where $\mathbf{H}=\left[H_{1}, H_{2}, \ldots, H_{n}\right]^{\mathrm{T}}$, and the drift coefficients $m_{i}(\mathbf{H} ; \boldsymbol{\tau})$ and diffusion coefficients $\bar{\sigma}_{i k}(\mathbf{H} ; \boldsymbol{\tau})$ are given by

$$
\begin{gathered}
m_{i}(\mathbf{H} ; \tau)=\frac{1}{T(\mathbf{H} ; \tau)} \\
. \oint\left(-\sum_{u=1,2}\left[c_{r j}^{\prime(u)}(\mathbf{q}, \mathbf{p} ; \tau) \cdot \mu^{(u)}\right] \frac{\partial H}{\partial p_{j}} \frac{\partial H_{i}}{\partial p_{r}}\right. \\
\left.+\frac{1}{2} \sigma_{r k} \sigma_{j k} \frac{\partial^{2} H_{i}}{\partial p_{r} \partial p_{j}}\right) \prod_{s=1}^{n}\left(\frac{\partial H_{s}}{\partial p_{s}}\right)^{-1} d q_{s} \\
b_{i s}(\mathbf{H} ; \tau)=\bar{\sigma}_{i k} \bar{\sigma}_{s k}(\mathbf{H} ; \tau)=\frac{1}{T(\mathbf{H} ; \tau)} \\
\cdot \oint\left(\sigma_{j k} \sigma_{l k} \frac{\partial H_{i}}{\partial p_{j}} \frac{\partial H_{s}}{\partial p_{l}}\right) \prod_{s=1}^{n}\left(\frac{\partial H_{s}}{\partial p_{s}}\right)^{-1} d q_{s}
\end{gathered}
$$

where $\oint \cdot d q_{s}$ represents an $n$-fold loop integral and

$$
T(\mathbf{H} ; \tau)=\oint \prod_{s=1}^{n}\left(\frac{\partial H_{s}}{\partial p_{s}}\right)^{-1} d q_{s}
$$

After this approximation, the dimension of system is reduced significantly from $2 n$ to $n$.

\section{The Largest Lyapunov Exponent}

Linearizing (13) at $\mathbf{H}=0$ yields

$$
d H_{i}=m_{i}^{L}(\mathbf{H} ; \tau) d t+\bar{\sigma}_{i k}^{L}(\mathbf{H} ; \tau) d B_{k}(t) .
$$


Since $(5 \mathrm{a})$ and $(5 \mathrm{~b})$ contain only parametric excitations, $\mathbf{Q}=$ $\mathbf{P}=\mathbf{0}$ is the trivial solution of (1) and $\mathbf{H}=\mathbf{0}$ is the trivial solution of (16). Then, the coefficients of (16) satisfy

$$
\begin{aligned}
\lim _{|\mathbf{H}| \rightarrow 0} m_{i}^{L}(\mathbf{H} ; \tau) & =0, \\
\lim _{|\mathbf{H}| \rightarrow 0} \bar{\sigma}_{i k}^{L}(\mathbf{H} ; \tau) & =0, \\
k m_{i}^{L}(\mathbf{H} ; \tau) & =m_{i}^{L}(k \mathbf{H} ; \tau), \\
k \bar{\sigma}_{i k}^{L}(\mathbf{H} ; \tau) & =\bar{\sigma}_{i k}^{L}(k \mathbf{H} ; \tau),
\end{aligned}
$$

$k>0$.

Introduce the following new variables:

$$
\begin{aligned}
\rho & =\frac{1}{2} \ln H, \\
\alpha_{i} & =\frac{H_{i}}{H}, \quad i=1,2, \ldots, n .
\end{aligned}
$$

The Itô equations for $\rho$ and $\alpha_{i}$ are then obtained from (16) by using Itô differential rule as follows:

$$
\begin{aligned}
d \rho & =G(\boldsymbol{\alpha} ; \tau) d t+\Xi_{u}(\boldsymbol{\alpha} ; \tau) d B_{u}(t), \\
d \alpha_{i} & =\bar{G}_{i}(\boldsymbol{\alpha} ; \tau) d t+\bar{\Xi}_{i u}(\boldsymbol{\alpha} ; \tau) d B_{u}(t),
\end{aligned}
$$

where $\boldsymbol{\alpha}=\left[\alpha_{1}, \alpha_{2}, \ldots, \alpha_{n}\right]^{\mathrm{T}}$ and

$$
\begin{aligned}
G(\boldsymbol{\alpha} ; \tau)= & \frac{1}{2} \sum_{i=1}^{n} m_{i}^{L}(\boldsymbol{\alpha} ; \tau) \\
& -\frac{1}{4} \sum_{i, j=1}^{n} \sum_{k=1}^{m} \bar{\sigma}_{i k}^{L}(\boldsymbol{\alpha} ; \tau) \bar{\sigma}_{j k}^{L}(\boldsymbol{\alpha} ; \tau), \\
\bar{G}_{i}(\boldsymbol{\alpha} ; \tau)= & m_{i}^{L}(\boldsymbol{\alpha} ; \tau)-\alpha_{i} \sum_{s=1}^{n} m_{s}^{L}(\boldsymbol{\alpha} ; \tau) \\
& +\frac{1}{2} \alpha_{i} \sum_{r, j=1}^{n} \sum_{k=1}^{m} \bar{\sigma}_{r k}^{L}(\boldsymbol{\alpha} ; \tau) \bar{\sigma}_{j k}^{L}(\boldsymbol{\alpha} ; \tau) \\
& -\frac{1}{2} \sum_{j=1}^{n} \sum_{k=1}^{m} \bar{\sigma}_{i k}^{L}(\boldsymbol{\alpha} ; \tau) \bar{\sigma}_{j k}^{L}(\boldsymbol{\alpha} ; \tau), \\
\bar{\Xi}_{i u}(\boldsymbol{\alpha} ; \tau)= & \bar{\sigma}_{i u}^{L}(\boldsymbol{\alpha} ; \tau)-\alpha_{i} \sum_{j=1}^{n} \bar{\sigma}_{j u}^{L}(\boldsymbol{\alpha} ; \tau) .
\end{aligned}
$$

Define the Lyapunov exponent of system (16) as the asymptotic rate of exponential growth of the square-root of the Hamiltonian; that is,

$$
\Lambda=\lim _{t \rightarrow \infty} \frac{1}{t} \log H^{1 / 2} .
$$

Integrating (19a) from 0 to $t$ and then dividing it by $t$ yield

$$
\begin{aligned}
\frac{1}{2 t} \log H(t)= & \frac{1}{2 t} \log H(0)+\frac{1}{t} \int_{0}^{t} G(\boldsymbol{\alpha} ; \tau) d s \\
& +\frac{1}{t} \int_{0}^{t} \Xi_{u}(\boldsymbol{\alpha} ; \tau) d B_{u}(s) .
\end{aligned}
$$

As $t \rightarrow \infty$, the first and the last terms in the right hand side of (22) vanish and, as a consequence,

$$
\Lambda=\lim _{t \rightarrow \infty} \frac{1}{t} \int_{0}^{t} G(\boldsymbol{\alpha} ; \tau) d \tau
$$

From (18b), it is seen that $\sum_{i=1}^{n} \alpha_{i}=1$, and only $n-1$ equations for $\alpha_{i}$ in (19b) are independent. Without loss of generality, in the following, the first $(n-1)$ equations for $\alpha_{1}, \alpha_{2}, \ldots, \alpha_{n-1}$ are taken as independent equations. Let $\boldsymbol{\alpha}^{\prime}=\left[\alpha_{1}, \alpha_{2}, \ldots, \alpha_{n-1}\right]$ be $n-1$ dimensional vector diffusion process. Suppose that $\boldsymbol{\alpha}^{\prime}$ is ergodic over the interval $0 \leq \alpha_{k} \leq 1(k=1,2, \ldots, n-$ $1)$. Under this condition, the time averaging in (23) can be replaced by assembly averaging; that is,

$$
\Lambda=E\left[G\left(\boldsymbol{\alpha}^{\prime} ; \tau\right)\right]=\int G\left(\boldsymbol{\alpha}^{\prime} ; \tau\right) p\left(\boldsymbol{\alpha}^{\prime}\right) d \boldsymbol{\alpha}^{\prime},
$$

where $p\left(\boldsymbol{\alpha}^{\prime}\right)$ is the stationary probability density of vector process $\boldsymbol{\alpha}^{\prime}$, which can be obtained by solving the FokkerPlanck-Kolmogorov (FPK) equation associated with first $n-1$ independent equations of (19b).

For an $n$-dimensional (16), there exist $n$ Lyapunov exponents. However, (24) has been proved to converge to the largest Lyapunov exponent $\Lambda_{\max }$ [16]. Based on Oseledec ergodic theorem, a necessary and sufficient condition for asymptotic stability with probability one of the trivial solution of the system governed by (5a) and (5b) is approximately

$$
\Lambda_{\max }=\int G\left(\boldsymbol{\alpha}^{\prime} ; \tau\right) p\left(\boldsymbol{\alpha}^{\prime}\right) d \boldsymbol{\alpha}^{\prime}<0
$$

\section{Numerical Example}

To demonstrate the application and validity of the proposed method, consider a stochastically excited nonlinear twodegree system with time delay feedback and actuator failure, governed by

$$
\begin{aligned}
\dot{q}_{1}= & p_{1}, \\
\dot{p}_{1}= & -\omega_{1}^{2} q_{1}-\beta_{11} p_{1}-\beta_{22} p_{2}+\kappa^{[s(t)]} u(t-\tau) \\
& +f_{11} q_{1} \xi_{1}(t)+f_{12} q_{2}^{2} \xi_{2}(t), \\
\dot{q}_{2}= & p_{2}, \\
\dot{p}_{2}= & -\alpha q_{2}^{3}-\beta_{21} p_{1}-\beta_{22} p_{2}+f_{21} q_{1} \xi_{1}(t) \\
& +f_{22} q_{2}^{2} \xi_{2}(t),
\end{aligned}
$$

where $\omega_{1}, \alpha>0$, and $\beta_{i j}>0$ are constants; $\xi_{i}(t)$ is Gaussian white noise with zero mean and intensity $2 D ; \kappa^{[s(t)]} u(t-\tau)$ denotes the possible failure actuator with time delay. $\kappa^{[s(t)]}$ is the failure factor. The two-state Markov process $s(t)$ denotes the state of the actuator: $s(t)=1$ is the "failure" state and $s(t)=2$ is the "functional" state. $\lambda_{1}$ denotes the transition rate from the breakdown to the functional state and $\lambda_{2}$ backward transition state. The transition matrix $\Lambda=\left[\lambda_{i j}\right]$ has the form 
of (3). The stationary distribution for $s(t)=u$ is calculated from (4):

$$
\mu^{(u)}=\frac{\sum_{i=1 \neq u} \lambda_{i}}{\sum_{i=1,2} \lambda_{i}}, \quad u \in S=\{1,2\} .
$$

$u(t-\tau)$ can be specified as

$$
u(t-\tau)=-f_{1} q_{1}(t-\tau)-f_{2} p_{1}(t-\tau)
$$

According to the approximate expression as (9), (26) becomes

$$
\begin{aligned}
\dot{q}_{1}= & p_{1}, \\
\dot{p}_{1}= & -\sum_{u=1,2}\left[\left(\widetilde{\omega}_{1}^{(u)}\right)^{2} \mu^{(u)}\right] q_{1}-\sum_{u=1,2}\left[\widetilde{\beta}_{11}^{(u)} \mu^{(u)}\right] p_{1} \\
& -\beta_{22} p_{2}+f_{11} q_{1} \xi_{1}(t)+f_{12} q_{2}^{2} \xi_{2}(t), \\
\dot{q}_{2}= & p_{2}, \\
\dot{p}_{2}= & -\alpha q_{2}^{3}-\beta_{21} p_{1}-\beta_{22} p_{2}+f_{21} q_{1} \xi_{1}(t) \\
& +f_{22} q_{2}^{2} \xi_{2}(t),
\end{aligned}
$$

where

$$
\begin{aligned}
\left(\widetilde{\omega}_{1}^{(u)}\right)^{2} & =\omega_{1}^{2}+\left[f_{1} \cos \left(\omega_{1} \tau\right)+f_{2} \omega_{1} \sin \left(\omega_{1} \tau\right)\right] \kappa^{(u)}, \\
\tilde{\beta}_{11}^{(u)} & =\beta_{11}+\left[-\frac{f_{1} \sin \left(\omega_{1} \tau\right)}{\omega_{1}}+f_{2} \cos \left(\omega_{1} \tau\right)\right] \kappa^{(u)} .
\end{aligned}
$$

Note that the Hamiltonian system associated with (29) is completely integrable. The associated Hamiltonian is

$$
\begin{aligned}
H(\tau) & =\sum_{i=1,2} H_{i}\left(q_{i}, p_{i} ; \tau\right), \\
H_{1}\left(q_{1}, p_{1} ; \tau\right) & =\frac{1}{2} p_{1}^{2}+\frac{1}{2} \sum_{u=1,2}\left[\left(\widetilde{\omega}_{1}^{(u)}\right)^{2} \cdot \mu^{(u)}\right] q_{1}^{2}, \\
H_{2}\left(q_{2}, p_{2} ; \tau\right) & =\frac{1}{2} p_{2}^{2}+\frac{1}{4} \alpha q_{2}^{2} .
\end{aligned}
$$

After the limit averaging and stochastic averaging, the averaged Itô equation for system (29) can be derived as the form of (13). Linearizing the averaged Itô equation yields

$$
d H_{i}=m_{i}^{L}\left(H_{1}, H_{2} ; \tau\right) d t+\bar{\sigma}_{i k}^{L}\left(H_{1}, H_{2} ; \tau\right) d B_{k}(t),
$$

where

$$
\begin{aligned}
m_{1}^{L}\left(H_{1}, H_{2}\right)= & -\sum_{u=1,2}\left[\widetilde{\beta}_{11}^{(u)} \mu^{(u)}\right] H_{1} \\
& +\frac{f_{11}^{2} D_{1}}{\sum_{u=1,2}\left[\left(\widetilde{\omega}_{1}^{(u)}\right)^{2} \mu^{(u)}\right]} H_{1} \\
& +\eta_{1} f_{12}^{2} D_{2} H_{2}
\end{aligned}
$$

$$
m_{2}^{L}\left(H_{1}, H_{2}\right)=-\eta_{2} \beta_{22} H_{2}
$$

$$
\begin{aligned}
& +\frac{f_{21}^{2} D_{1}}{\sum_{u=1,2}\left[\left(\widetilde{\omega}_{1}^{(u)}\right)^{2} \mu^{(u)}\right]} H_{1} \\
& +\eta_{1} f_{22}^{2} D_{2} H_{2},
\end{aligned}
$$

$$
\begin{aligned}
& \bar{\sigma}_{1 k}^{L} \bar{\sigma}_{1 k}^{L}\left(H_{1}, H_{2}\right)=\frac{f_{11}^{2} D_{1}}{\sum_{u=1,2}\left[\left(\widetilde{\omega}_{1}^{(u)}\right)^{2} \mu^{(u)}\right]} H_{1}^{2}, \\
& \bar{\sigma}_{2 k}^{L} \bar{\sigma}_{2 k}^{L}\left(H_{1}, H_{2}\right)=2 \eta_{3} f_{22}^{2} D_{2} H_{2}^{2}, \\
& \bar{\sigma}_{1 k}^{L} \bar{\sigma}_{2 k}^{L}\left(H_{1}, H_{2}\right)=0,
\end{aligned}
$$

where

$$
\begin{aligned}
& \eta_{1}=\frac{(8 / 9 \alpha)^{1 / 2}}{\int_{0}^{1}\left(1-t^{4}\right)^{-1 / 2} d t}, \\
& \eta_{2}=2 \frac{\int_{0}^{1}\left(1-t^{4}\right)^{1 / 2} d t}{\int_{0}^{1}\left(1-t^{4}\right)^{-1 / 2} d t}, \\
& \eta_{3}=\frac{(8 / \alpha) \int_{0}^{1} t^{4}\left(1-t^{4}\right)^{1 / 2} d t}{\int_{0}^{1}\left(1-t^{4}\right)^{-1 / 2} d t} .
\end{aligned}
$$

Note that the drift and diffusion coefficients in (33) are homogeneous in $H_{i}$ of order one. Introducing the transformation as the form in (18a) and (18b), the Itô equations for $\rho$ and $\alpha_{1}$ are obtained

$$
\begin{aligned}
d \rho & =G\left(\alpha_{1} ; \tau\right) d t+\Xi\left(\alpha_{1} ; \tau\right) d B(t), \\
d \alpha_{1} & =\bar{G}\left(\alpha_{1} ; \tau\right) d t+\bar{\Xi}\left(\alpha_{1} ; \tau\right) d B(t),
\end{aligned}
$$

where

$$
\begin{aligned}
G\left(\alpha_{1}\right)= & \left(\mu_{1}+\frac{f_{21}^{2} D_{1}}{\sum_{u=1,2}\left[\left(\widetilde{\omega}_{1}^{(u)}\right)^{2} \mu^{(u)}\right]}\right) \alpha_{1} \\
& +\left(\mu_{2}+f_{12}^{2} D_{2} \eta_{1}\right)\left(1-\alpha_{1}\right) \\
& +\frac{A \alpha_{1}\left(1-\alpha_{1}\right)}{4}, \\
\bar{G}\left(\alpha_{1}\right)= & {\left[2\left(\mu_{1}-\mu_{2}\right)+\frac{A\left(1-2 \alpha_{1}\right)}{2}\right] \alpha_{1}\left(1-\alpha_{1}\right) } \\
& +f_{12}^{2} D_{2} \eta_{1}\left(1-\alpha_{1}\right)^{2} \\
& -\frac{f_{21}^{2} D_{1}}{\sum_{u=1,2}\left[\left(\widetilde{\omega}_{1}^{(u)}\right)^{2} \mu^{(u)}\right]} \alpha_{1}^{2},
\end{aligned}
$$




$$
\begin{aligned}
\bar{\Xi}^{2}\left(\alpha_{1}\right) & =A \alpha_{1}^{2}\left(1-\alpha_{1}\right)^{2}, \\
\mu_{1} & =-\frac{1}{2} \sum_{u=1,2}\left[\widetilde{\beta}_{11}^{(u)} \mu^{(u)}\right]+\frac{f_{11}^{2} D_{1}}{\sum_{u=1,2}\left[\left(\widetilde{\omega}_{1}^{(u)}\right)^{2} \mu^{(u)}\right]}, \\
\mu_{2} & =-\frac{1}{2} \beta_{22} \eta_{2}+\frac{1}{2}\left(\eta_{1}-\eta_{3}\right) f_{22}^{2} D_{2} \\
A & =\frac{f_{11}^{2} D_{1}}{\sum_{u=1,2}\left[\left(\widetilde{\omega}_{1}^{(u)}\right)^{2} \mu^{(u)}\right]}+2 \eta_{3} f_{22}^{2} D_{2} .
\end{aligned}
$$

Following (24), the largest Lyapunov exponent is then obtained

$$
\Lambda_{\max }=\int_{0}^{1} G\left(\alpha_{1} ; \tau\right) p\left(\alpha_{1}\right) d \alpha_{1} .
$$

The stationary probability density $p\left(\alpha_{1}\right)$ can be obtained by solving the Fokker-Planck-Kolmogorov (FPK) equation associated with Itô equation for $\alpha_{1}$ as

$$
\begin{gathered}
p\left(\alpha_{1}\right)=\frac{C}{\alpha_{1}\left(1-\alpha_{1}\right)}\left(\frac{\alpha_{1}}{1-\alpha_{1}}\right)^{4\left(\mu_{1}-\mu_{2}\right) / A} \\
\quad \times \exp \left\{-\frac{2}{A}\left[\frac{\eta_{1} f_{12}^{2} D_{2}}{\alpha_{1}}\right.\right. \\
\left.\left.+\frac{f_{21}^{2} D_{1}}{\left(1-\alpha_{1}\right) \sum_{u=1,2}\left[\left(\widetilde{\omega}_{1}^{(u)}\right)^{2} \mu^{(u)}\right]}\right]\right\} .
\end{gathered}
$$

Some numerical results are obtained as shown in Figures 1-4 for system parameters

$$
\begin{aligned}
& \beta_{11}=0.01 ; \\
& \beta_{12}=0.0 ; \\
& \beta_{21}=0.0 ; \\
& \beta_{22}=0.01 ; \\
& f_{11}=1.0 ; \\
& f_{12}=1.0 ; \\
& f_{21}=1.0 ; \\
& f_{22}=1.0 ; \\
& \omega_{1}=3.0 ; \\
& \alpha=0.2 ; \\
& f_{1}=0.04 ; \\
& f_{2}=0.04 ; \\
& D_{1}=0.01 ; \\
& D_{2}=0.01 ;
\end{aligned}
$$

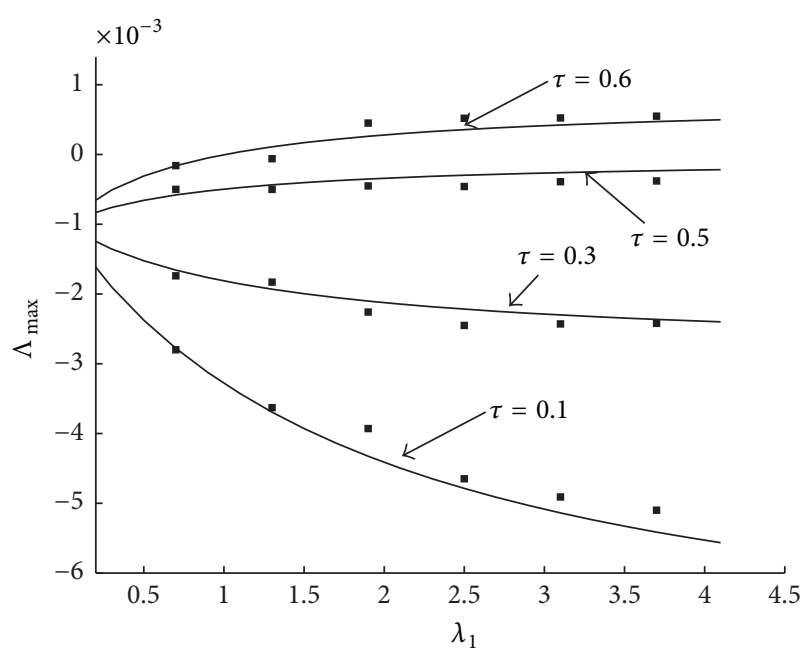

Figure 1: The largest Lyapunov exponent $\Lambda_{\max }$ as function of transition rate $\lambda_{1}$. The lines are obtained from numerical solution of (37) while the symbols are obtained from direct simulation of original system (26).

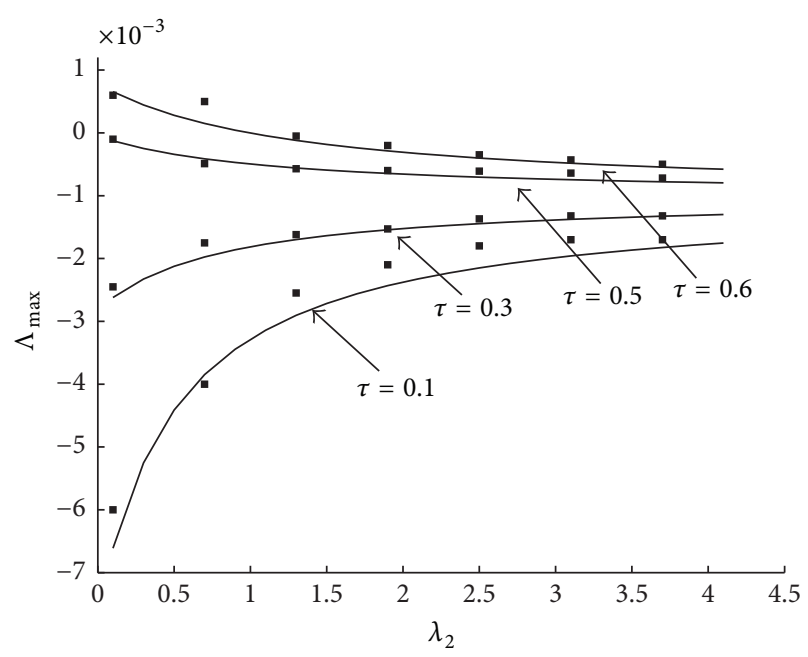

Figure 2: The largest Lyapunov exponent $\Lambda_{\max }$ as function of transition rate $\lambda_{2}$. The lines are obtained from numerical solution of (37) while the symbols are obtained from direct simulation of original system (26).

$$
\begin{aligned}
& \kappa^{(1)}=0.0 ; \\
& \kappa^{(2)}=1.0 .
\end{aligned}
$$

Figure 1 shows the largest Lyapunov exponent $\Lambda_{\max }$ as function of transition rate $\lambda_{1}$. It is seen that, for small delays, the largest Lyapunov exponent $\Lambda_{\max }$ is a monotonous decreasing function of $\lambda_{1}$. This is understandable: for small delay, the feedback control plays positive role in stability of system (26). As $\lambda_{1}$ increases, the failure probability of the actuator decreases, and the stability of the system is enhanced as a result. However, for large delay, $\Lambda_{\max }$ is a monotonous increasing function of $\lambda_{1}$, as also shown in 


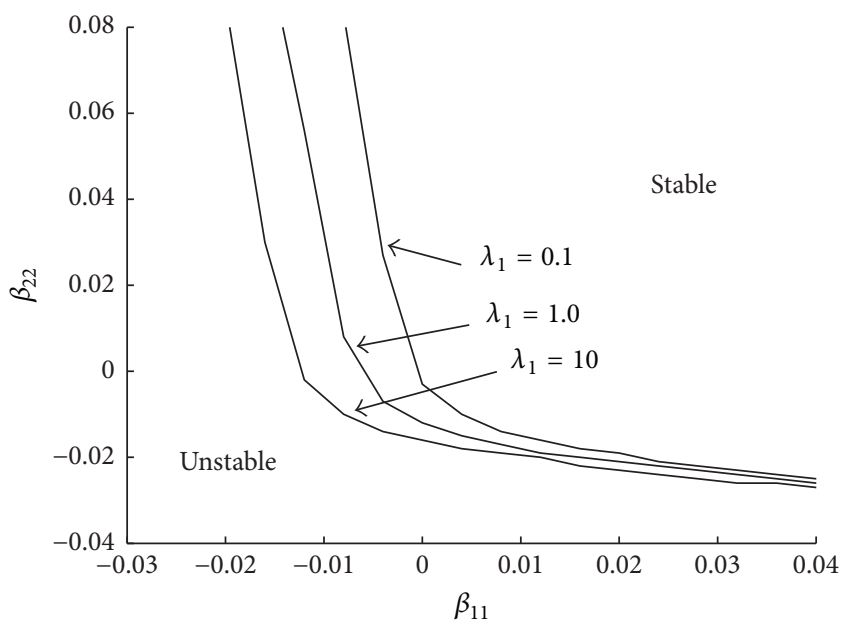

FIGURE 3: The stability boundaries in the $\left(\beta_{11}, \beta_{22}\right)$ plane for different transition rate $\lambda_{1}$ when $\tau=0.3$.

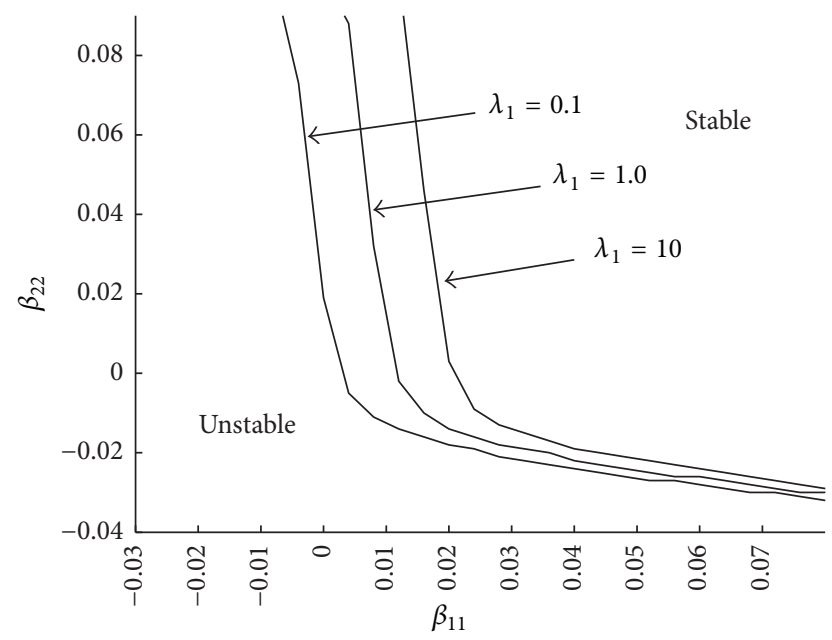

FIgURE 4 : The stability boundaries in the $\left(\beta_{11}, \beta_{22}\right)$ plane for different transition rate $\lambda_{1}$ when $\tau=0.6$.

Figure 1. This implies that higher probability of actuator working in functional state deteriorates the performance of the dynamics. This is because that large delay feedback control brings negative damping to the system, as shown in (30), which has negative contribution to the stability of system. In Figure 2, the variation of $\Lambda_{\max }$ with $\lambda_{2}$ is displayed for different time delay. It is seen that as $\lambda_{2}$ increases, $\Lambda_{\max }$ increases for small delay while decreasing for large delay. The observation can be explained in a similar fashion as in Figure 1.

In Figure 3 , the stability boundaries in the $\left(\beta_{11}, \beta_{22}\right)$ plane for different transition rate $\lambda_{1}$ in the small delay case are constructed by imposing $\Lambda_{\max }=0$. In small delay case, as $\lambda_{1}$ is increased, the stability region of the system increases. Figure 4 shows the stability boundaries in $\left(\beta_{11}, \beta_{22}\right)$ plane for different transition rate $\lambda_{1}$ in the large delay case. Obviously, in large time delay case, large $\lambda_{1}$ will reduce the stability region of the system.
To verify the largest Lyapunov exponent calculated by using (37), the direct simulation of (26) is also obtained and shown in Figures 1 and 2 by using symbols $\mathbf{m}$. Observe that the symbols match closely with the solid lines, demonstrating the validity and accuracy of the proposed procedure.

\section{Conclusions}

In this paper, the asymptotic stability with probability of one of delay-controlled nonlinear systems with random actuator failures was investigated. Based on the random switch model of failure-prone actuator, the stability problem of such a system has been converted into the framework of Markov jump hybrid systems. Upon the limit principle and stochastic averaging, an approximate expression for the largest Lyapunov exponent of the original system was derived. Important results reported in the paper are summarized in the following statements:

(1) Actuator failure has significant effects on the asymptotic stability of the delay-controlled system. For small delay, high probability of actuator failure will degrade the stability while enhancing the stability for large delay.

(2) The validity and utility of the propose procedure have been demonstrated by using a delay-controlled nonlinear two-degree system with actuator failure. By comparison with direct system simulations, it has been observed that the procedure is fairly reliable in assessing asymptotic stability.

\section{Disclosure}

Opinions, findings, and conclusions expressed in this paper are those of the authors and do not necessarily reflect the views of the sponsors.

\section{Conflicts of Interest}

The authors declare that there are no conflicts of interest regarding the publication of this paper.

\section{Acknowledgments}

This work was supported by the Natural Science Foundation of China through Grants nos. 11372271, 11432012, and 11621062 .

\section{References}

[1] Y. Zhang and X. R. Li, "Detection and diagnosis of sensor and actuator failures using IMM estimator," IEEE Transactions on Aerospace and Electronic Systems, vol. 34, no. 4, pp. 1293-1313, 1998.

[2] J. Wang and H. Shen, "Passivity-based fault-tolerant synchronization control of chaotic neural networks against actuator faults using the semi-Markov jump model approach," Neurocomputing, vol. 143, pp. 51-56, 2014. 
[3] M. Mahmoud, J. Jiang, and Y. Zhang, "Stochastic stability analysis of fault-tolerant control systems in the presence of noise," Institute of Electrical and Electronics Engineers. Transactions on Automatic Control, vol. 46, no. 11, pp. 1810-1815, 2001.

[4] A. N. Gundes, "Stability of feedback systems with sensor or actuator failures: analysis," International Journal of Control, vol. 56, no. 4, pp. 735-753, 1992.

[5] S. Aberkane, D. Sauter, J. C. Ponsart, and D. Theilliol, "Ho stochastic stabilization of active fault tolerant control systems: convex approach," in Proceedings of the 44th IEEE Conference on Decision and Control, and the European Control Conference, CDC-ECC '05, pp. 3783-3788, Seville, Spain, December 2005.

[6] G. Tao, S. Chen, and S. M. Joshi, "An adaptive control scheme for systems with unknown actuator failures," Automatica. A Journal of IFAC, the International Federation of Automatic Control, vol. 38, no. 6, pp. 1027-1034, 2002.

[7] C.-J. Seo and B. K. Kim, "Robust and reliable $H_{\infty}$ control for linear systems with parameter uncertainty and actuator failure," Automatica. A Journal of IFAC, the International Federation of Automatic Control, vol. 32, no. 3, pp. 465-467, 1996.

[8] H. Y. Hu and Z. H. Wang, Dynamics of controlled mechanical systems with delayed feedback, Springer Berlin Heidelberg, 2002.

[9] M. Di Paola and A. Pirrotta, "Time delay induced effects on control of linear systems under random excitation," Probabilistic Engineering Mechanics, vol. 16, no. 1, pp. 43-51, 2001.

[10] Z. H. Liu and W. Q. Zhu, "Stochastic averaging of quasiintegrable Hamiltonian systems with delayed feedback control," Journal of Sound and Vibration, vol. 299, no. 1-2, pp. 178-195, 2007.

[11] F. Tao and Q. Zhao, "Synthesis of active fault-tolerant control based on Markovian jump system models," IET Control Theory \& Applications, vol. 1, no. 4, pp. 1160-1168, 2007.

[12] R. H. Huan, R. C. Hu, D. Pu, and W. Q. Zhu, "Optimal vibration control of a class of nonlinear stochastic systems with Markovian jump," Shock and Vibration, vol. 2016, Article ID 9641075, 2016.

[13] R. H. Huan, W.-Q. Zhu, F. Ma, and Z.-G. Ying, "Stationary response of a class of nonlinear stochastic systems undergoing Markovian jumps," Journal of Applied Mechanics, vol. 82, no. 5, Article ID 051008, 6 pages, 2015.

[14] W. Q. Zhu, "Nonlinear stochastic dynamics and control in Hamiltonian formulation," Applied Mechanics Reviews, vol. 59, no. 4, pp. 230-248, 2006.

[15] A. V. Skorokhod, Asymptotic Methods in the Theory of Stochastic Differential Equations, American Mathematical Society, Providence, RI, USA, 1989.

[16] W. Q. Zhu and Z. L. Huang, "Lyapunov exponents and stochastic stability of quasi-integrable-Hamiltonian systems," American Society of Mechanical Engineers. Transactions of the ASME. Journal of Applied Mechanics, vol. 66, no. 1, pp. 211-217, 1999. 


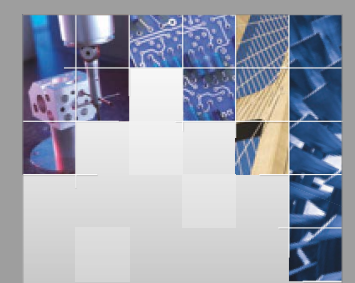

\section{Enfincering}
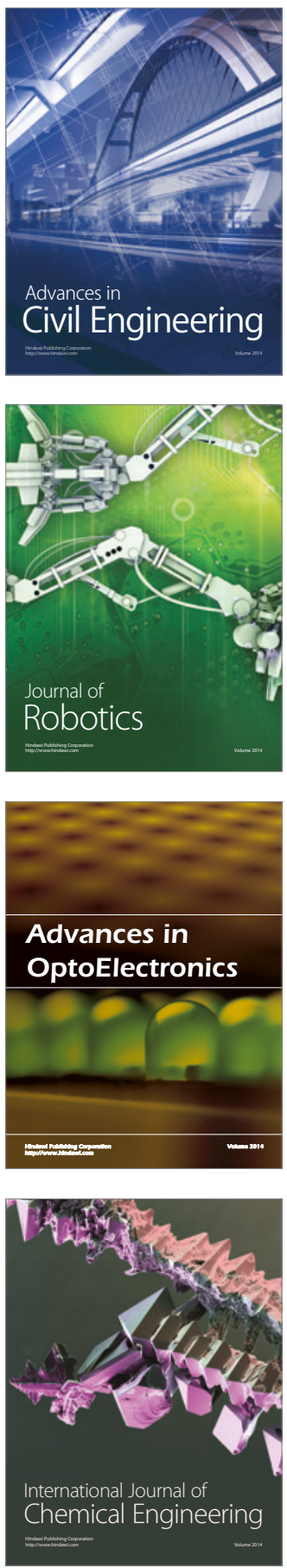

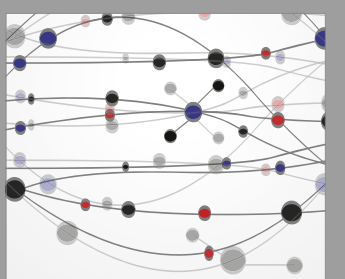

The Scientific World Journal

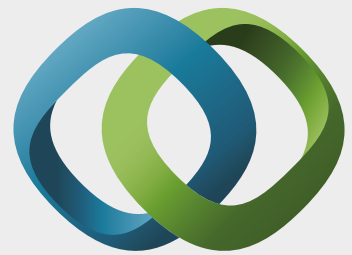

\section{Hindawi}

Submit your manuscripts at

https://www.hindawi.com
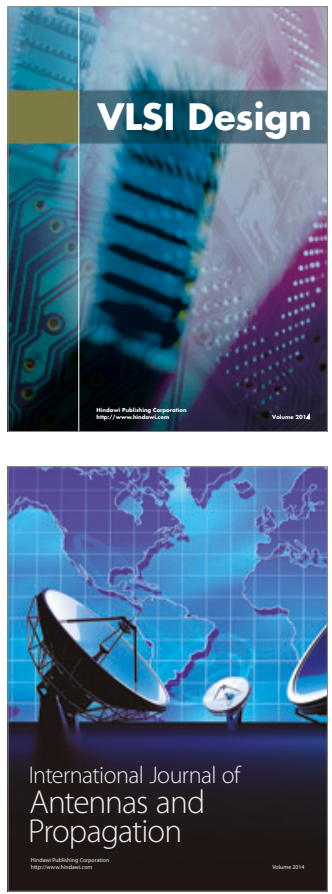

\section{Rotating}

Machinery
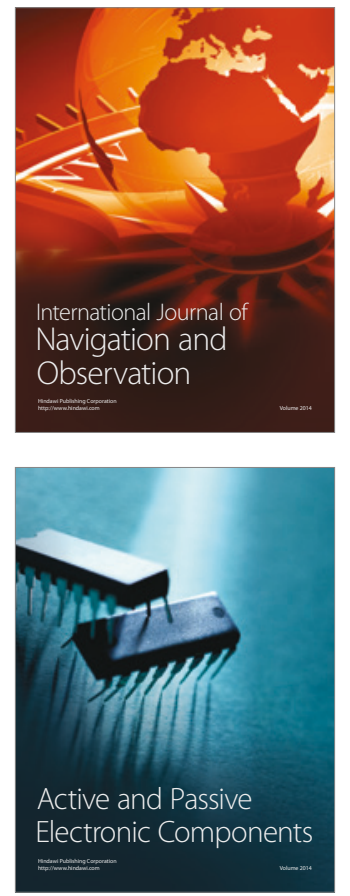
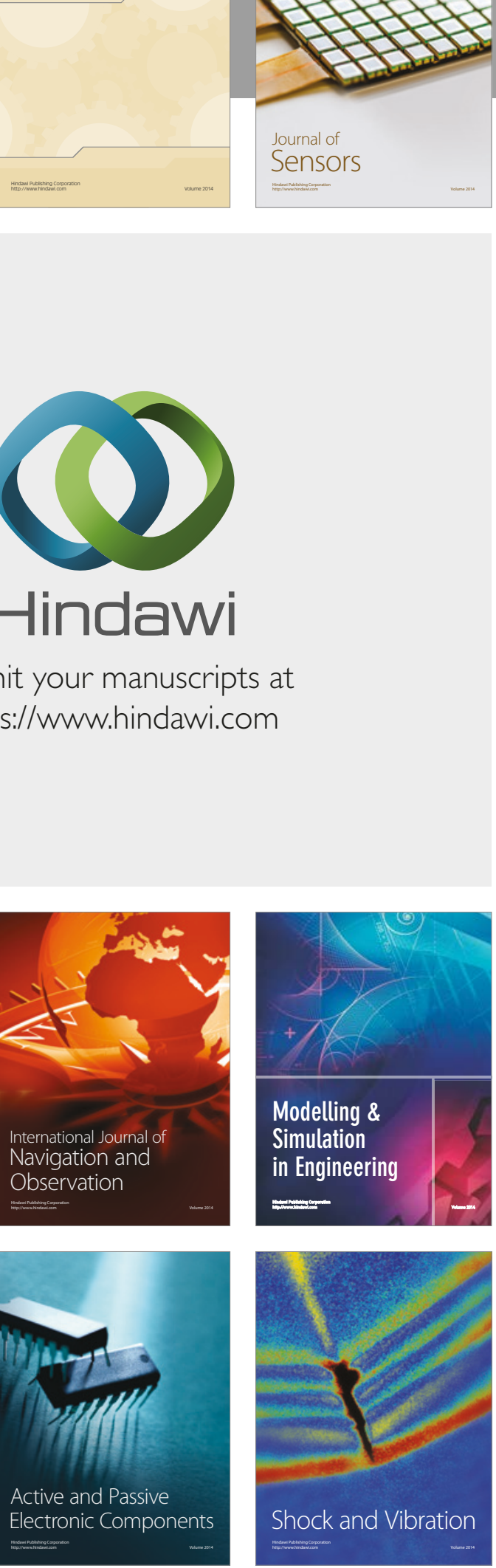
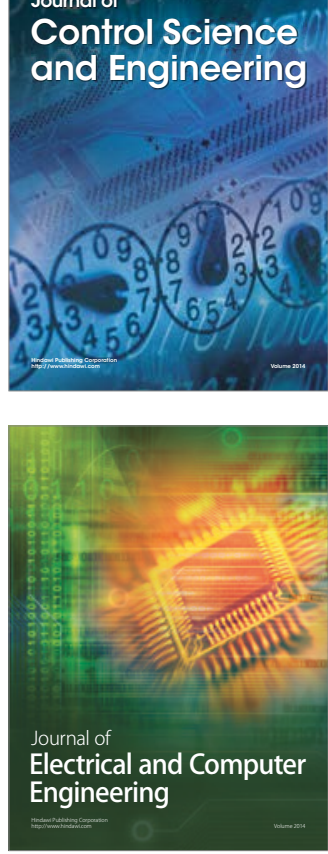

Distributed

Journal of

Control Science

and Engineering
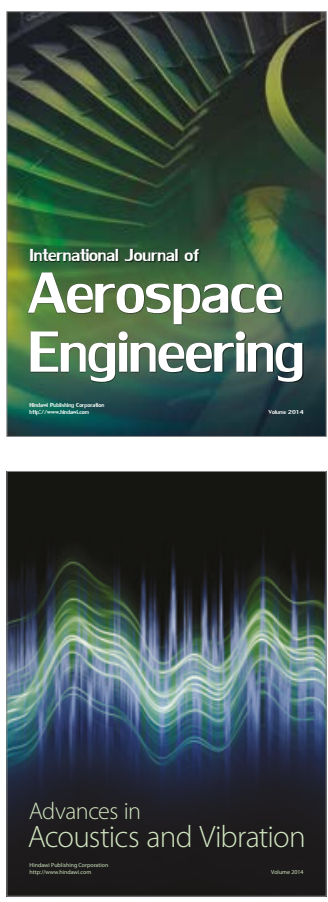

Sensor Networks 